\title{
Parametric Synthesis of Meander Slow-Wave System with Additional Shields
}

\author{
E. Metlevskis*, R. Martavičius, A. Katkevičius, D. Plonis and A. Krukonis \\ Vilnius Gediminas Technical University, Department of Electronic System, \\ Naugarduko Str. 41-413, LT-03227, Vilnius, Lithuania \\ (Received August 17, 2016; in final form November 3, 2016)
}

\begin{abstract}
The algorithm of parametric synthesis of meander slow-wave system with additional shields is presented in this paper. The influence of main constructive parameters of meander slow-wave system with additional shields on its electrical characteristics was evaluated in order to achieve the optimal results of the synthesis. Verification of the algorithm of parametric synthesis of meander slow-wave system with additional shields was carried out using four different models of meander slow-wave system with additional shields grounded in different positions. Sonnet ${ }^{\circledR}$ software package which is based on the method of moments has been used during the investigation.
\end{abstract}

DOI: 10.12693/APhysPolA.130.1435

PACS/topics: 84.40.Az, 84.40.Lj

\section{Introduction}

Meander slow-wave systems (MSSs) are planar structures, which can be manufactured using standard technologies, which are suitable for manufacturing of integrated microwaves circuits. Pass-band (PB) of such systems begins in the low frequencies therefore these systems are widely applied as delay lines and deflection systems [1-3]. Since MSSs provide compact size and high efficiency in wide frequency range, these systems find applications in antennas $[4,5]$, biomedicine [6], filters $[7,8]$, phase shifters [9].

Modern microwave devices, including slow-wave systems are designed using computer-aided design. The design process of microwave devices is carried out in several stages: analysis and synthesis of construction of the initial variant, adjustment of the initial variant and analysis of the new variant, optimization of construction, project documentation [10].

There is a need to create sophisticated constructions of meander systems with the development of microwave devices [11]. The design process of meander systems becomes more complex therefore more urgent task becomes the development of methods of computer-aided design [12].

It is important to select the mathematical model, synthesis and optimization methods of the system properly in order to minimize the cost of experimental research and design of meander slow-wave system.

The generalized computer-aided design process of MSSs is discussed in this article. The algorithm of parametric synthesis of MSS with additional shields grounded at both ends is formed after evaluation of influence of main constructive parameters of the system on its

\footnotetext{
* corresponding author; e-mail edvardas.metlevskis@gmail.com
}

electrical characteristics. Verification of the algorithm of parametric synthesis is carried out by performing synthesis of four different meander slow-wave systems.

\section{Model of the meander slow-wave system}

A meander slow-wave system is shown in Fig. 1. It consists of a conductor, which has a shape of meander. Conductor is placed on a dielectric substrate with the permittivity $\varepsilon_{\mathrm{r}}$ and a thickness $h$. Bottom of the substrate is covered by a conductive layer, which has a function of a grounded external shield. A single conductor has a length of $2 A$ and width $w$, a gap between neighboring conductors is $s$.

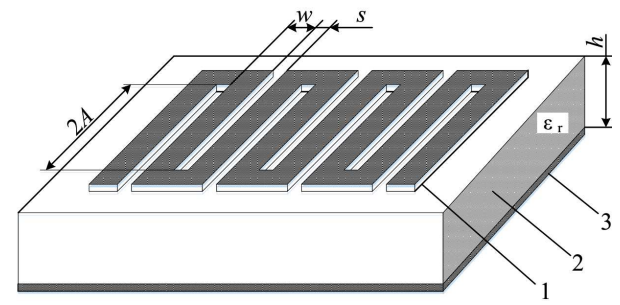

Fig. 1. Meander slow-wave system: 1 - meandershape conductor, 2 - dielectric substrate of relative permittivity $\varepsilon_{\mathrm{r}}, 3$ - external shield [14].

Broadband signals which propagate in the meander slow-wave system are distorted due to dispersion of phase delay time which is caused by electromagnetic coupling between adjacent meander strips which depend on the frequency. The additional shields are used in meander slow-wave system, which are fitted between the meander conductor strips in order to reduce the dispersion [10].

Additional shields are grounded via jumpers to an external shield, which as a rule is connected to the ground. Properties of MSS change when grounding place of external shield changes. 
Four models of MSSs, whose topologies are shown in Fig. 2, are created and investigated in order to improve the dispersive properties and increase a bandwidth of meander slow-wave system. Created models of meander slow-wave system differ in position of via along the additional shield (Fig. 2).
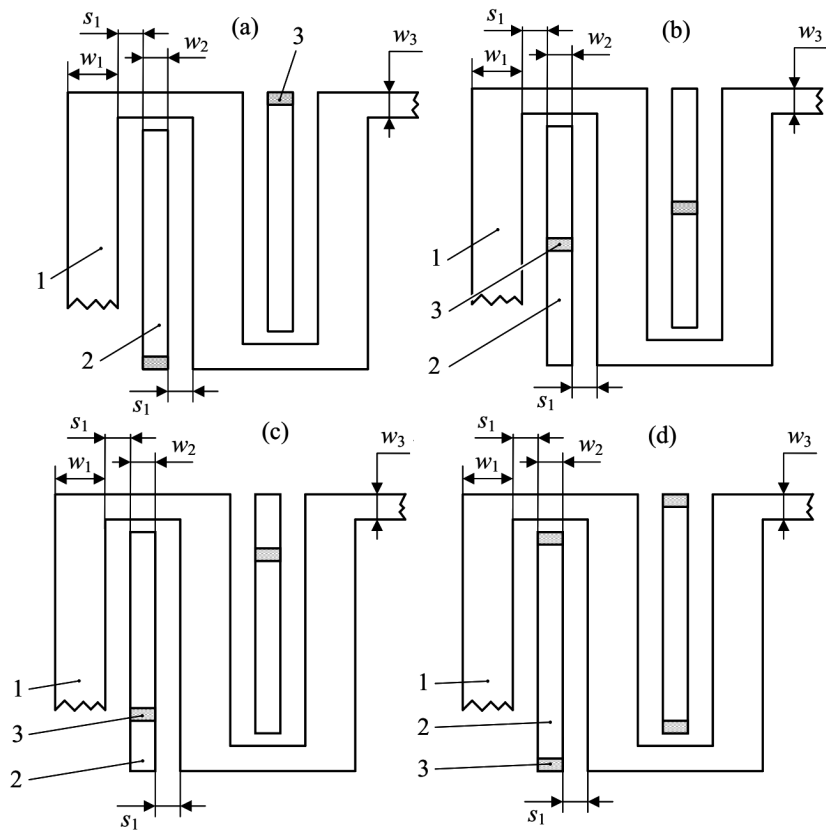

Fig. 2. Topology of MSS with additional shields grounded at: (a) one edge, (b) center, (c) point between the edge and center, (d) both edges; 1 - meander-shape conductor, 2 - additional shield, 3 - grounded via.

The width of meander-shape conductor is identified by $w_{1}$, the width of the additional shield is identified by $w_{2}$, the width of conductors which connect the adjacent strips is identified by $w_{3}$ and the width between the meander-shape conductor and additional shield is identified by $s_{1}$.

Models of MSSs with additional shields reflect changes in positions where additional shields are grounded to an external shield via jumpers. Additional shields are grounded at one end of the shield where there are no interconnections between meander strips in the first model (Fig. 2a). Jumpers are in the middle of additional shields in the second model (Fig. 2b). The MSSs with additional shields grounded with jumpers between the end and the middle point of the shield is shown in the third model (Fig. 2c). Additional shields are grounded at both ends in the fourth model (Fig. 2d).

Investigation of MSS with additional shields was performed using Sonnet ${ }^{\circledR}$ software package which is based on the method of moments. Slow-wave system has a rectangular shape; it consists of 7 parallel conductors which are connected in a shape of meander and 6 additional shields which are connected by vias at both ends to an external shield (Fig. 3).

Strips of a meander together with additional shields are placed on a dielectric plate of permittivity $\varepsilon_{\mathrm{r}}$. Bottom of

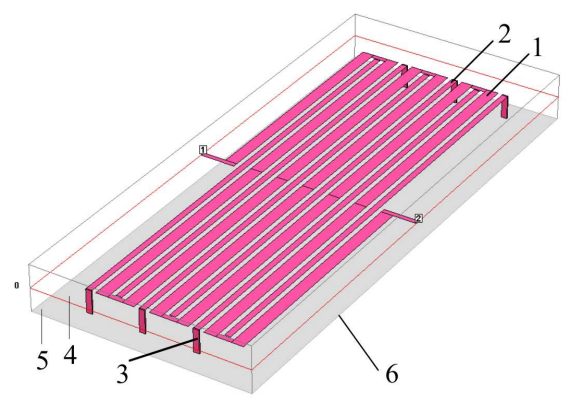

Fig. 3. 3D model of MSS with additional shields grounded at one edge: 1 - meander-shape conductor, 2 - additional shield, 3 - grounded via, 4 - air, 5 dielectric substrate, 6 - grounded external shield.

a plate is covered with metal, which forms an external shield. Above the dielectric plate is air. Therefore crosssection of a system contains two layers of dielectric. Bottom layer has a permittivity of $\varepsilon_{\mathrm{r}}=7.3$, which is a glass ceramic dielectric material used in the manufacture of microwave integrated circuits [13]. Top layer consists of air, which has a relative dielectric permittivity of 1 . Input and output of a system were placed at the center of meander. Initial dimensions of a meander slow-wave system are: $2 A=19.6 \mathrm{~mm}, w_{1}=0.5 \mathrm{~mm}, w_{2}=0.25 \mathrm{~mm}$, $w_{3}=0.2 \mathrm{~mm}, s_{1}=0.2 \mathrm{~mm}, h=0.5 \mathrm{~mm}$. Perfect conductors were used during the investigation. The system was investigated without losses.

\section{The analysis algorithm of parametric synthesis}

The algorithm of the parametric synthesis of MSS with additional shields grounded at both ends is shown in Fig. 4.

Parametric synthesis starts from the input of necessary electrical characteristics: width $\Delta F_{\text {need }}$ of pass-band, phase delay time $t_{\text {dneed }}$ and input impedance $Z_{\text {INneed }}$ (1 block). Designer selects the initial constructive parameters of the system ( 2 block) and synthesizes the initial variant of structure of MSS using his experience in the next stage of algorithm. This is done in iterative manner by managing the computer-aided design in MATLAB $^{\mathrm{TM}}$. Furthermore in this stage it is possible to modify number $N$ of meander conductors, relative permittivity $\varepsilon_{\mathrm{r}}$ and thickness $h$ of substrate whose change is not foreseen in later stages. The initial variant of the MSS is plotted by using SonnetLAb. The initial variant of the meander slow-wave system is analyzed with Sonnet ${ }^{\circledR}$ program with $\mathrm{em}^{\circledR}$ package, intended for analysis of high frequency planar circuits with method of moments afterwards.

The process of automatic synthesis begins after selection of the initial values of constructive parameters. The process of automatic synthesis consist of three cycles. Constructive parameter, which has the greatest impact on one of electrical characteristics, is replaced repeatedly until the required value of this electrical characteristic is received in the low frequencies. 


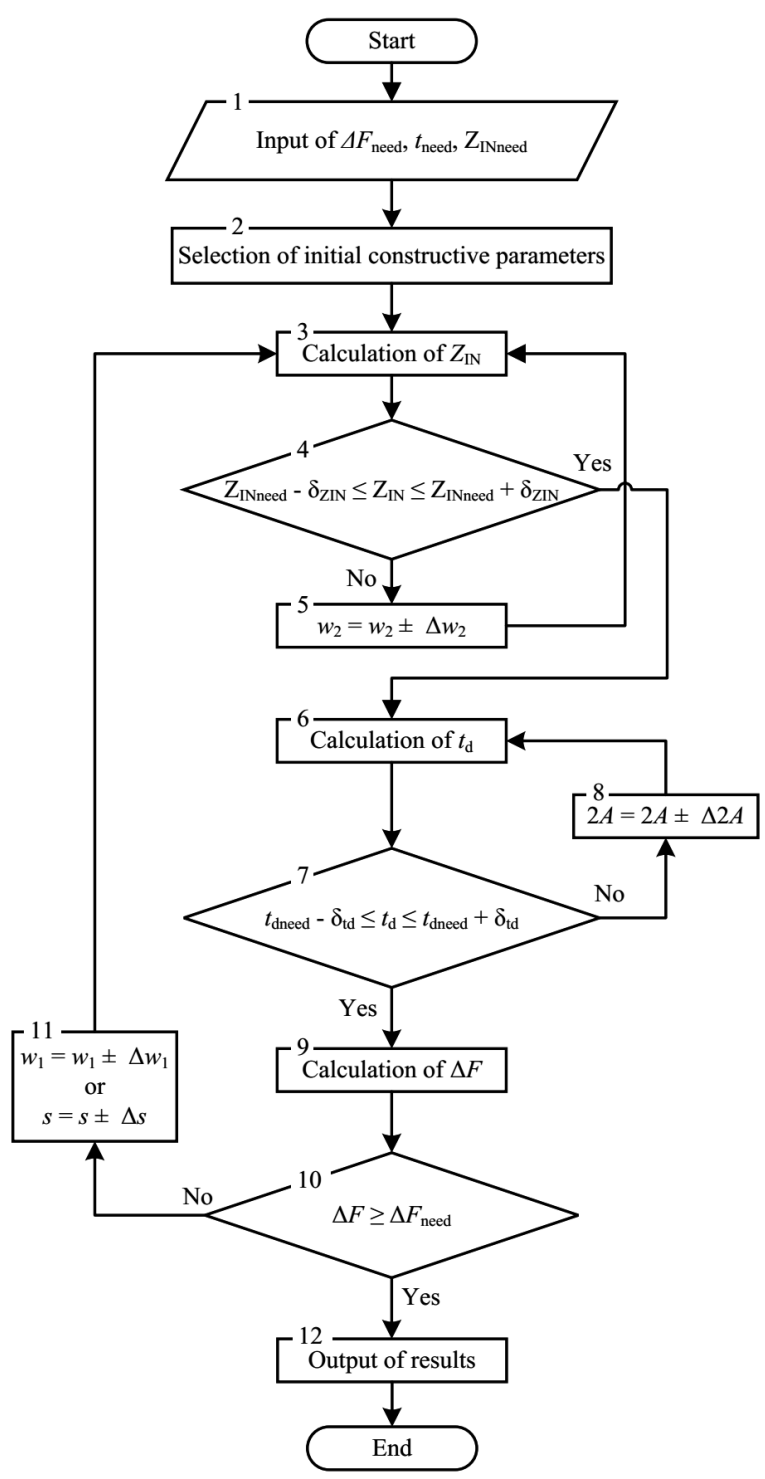

Fig. 4. Algorithm for parametric synthesis of a meander slow-wave system with additional shields grounded at both edges.

Sensitivity of electrical characteristics of the MSS with additional shields to variation of constructive parameters of the system was discussed in detail in the dissertation [14]. Values of other constructive parameters are fixed during this process.
The width $w_{2}$ of additional shield is repeatedly changed until the required value of the input impedance $Z_{\text {INneed }}$ is obtained in the low frequencies during first cycle (3-5 blocks). The length $2 A$ of the meander conductor is repeatedly changed as long as the necessary value of delay time $t_{\text {dneed }}$ is obtained in low frequencies in the second cycle $(6-8$ blocks $) . \delta_{\mathrm{IN}}$ and $\delta_{\mathrm{td}}$ parameters correspond the relative error of input impedance $Z_{\mathrm{IN}}$ and delay time $t_{\mathrm{d}}$, respectively ( 4 and 7 blocks).

The width $w_{1}$ of the meander conductor or the width $s$ of the gap between adjacent strips of meander that affect the width $\Delta F$ of the $\mathrm{PB}$ may be modified in the third cycle (10-11 blocks).

The parameter $\Delta F$ is the width of pass-band of phase delay time of meander slow-wave system with additional shields. However, the input impedance $Z_{\text {IN }}$ and delay time $t_{\mathrm{d}}$ of the system are changing as constructive parameters change. Therefore the algorithm returns to the first after the third cycle.

Characteristics which were calculated during the analysis are compared with the technical requirements. Parameters of the model are changed and the analysis procedure is repeated again if results of comparison are not satisfied. Parametric synthesis and optimization of the system is carried out in this case.

\section{Results of computer study}

Verification results including electrical characteristics and synthesized constructive parameters of four different meander slow-wave systems with additional shields are presented in Table I. Two different dielectric substrates were used during the synthesis. RO3006 $6^{\mathrm{TM}}$ material has a permittivity of $\varepsilon_{\mathrm{r}}=6.15$ and is made in Rogers Corporation and FR4 material has a permittivity of $\varepsilon_{\mathrm{r}}=4.4$ and is widely used in the manufacture of integrated circuit.

It can be seen from data presented in Table I that the bigger input impedance is obtained when the thickness $h$ of dielectric substrate and the width $w_{2}$ of additional shield increase and the width $w_{1}$ of meander electrode decrease at constant delay time $t_{\mathrm{d}}$ and width $\Delta F$ of $\mathrm{PB}$ (LS1 and LS2).

Three times bigger delay time $t_{\mathrm{d}}$ is obtained when the number of conductors $N$ increase three times, the length $2 A$ of meander conductor slightly increase and the width $w_{2}$ of additional shield decrease at constant $50 \Omega$ input impedance (LS1 and LS3). The width $\Delta F$ of PB decrease $33 \%$ because of these changes.

Electrical properties and constructive parameters of synthesized meander slow-wave system with grounded additional shields.

\begin{tabular}{c|c|c|c|c|c|c|c|c|c|c}
\hline \hline $\begin{array}{c}\text { Model of meander } \\
\text { slow-wave systems }\end{array}$ & $\begin{array}{c}t_{\mathrm{d}} \\
{[\mathrm{ns}]}\end{array}$ & $\begin{array}{c}Z_{\mathrm{IN}} \\
{[\Omega]}\end{array}$ & $\begin{array}{c}\Delta F \\
{[\mathrm{GHz}]}\end{array}$ & $\begin{array}{c}h \\
{[\mathrm{~mm}]}\end{array}$ & $\varepsilon_{\mathrm{r}}$ & $\begin{array}{c}w_{1} \\
{[\mathrm{~mm}]}\end{array}$ & $\begin{array}{c}w_{2} \\
{[\mathrm{~mm}]}\end{array}$ & $\begin{array}{c}s \\
{[\mathrm{~mm}]}\end{array}$ & $\begin{array}{c}2 A \\
{[\mathrm{~mm}]}\end{array}$ & $N$ \\
\hline LS1 & 1 & 50 & 2.72 & 0.13 & 6.15 & 0.22 & 0.47 & 0.9 & 25.0 & 5 \\
LS2 & 1 & 75 & 2.72 & 0.25 & 6.15 & 0.18 & 0.80 & 0.9 & 25.0 & 5 \\
LS3 & 3 & 50 & 1.82 & 0.13 & 6.15 & 0.22 & 0.40 & 0.9 & 25.5 & 15 \\
LS4 & 1 & 50 & 2.67 & 0.3 & 4.40 & 0.62 & 0.85 & 0.4 & 29.4 & 5
\end{tabular}




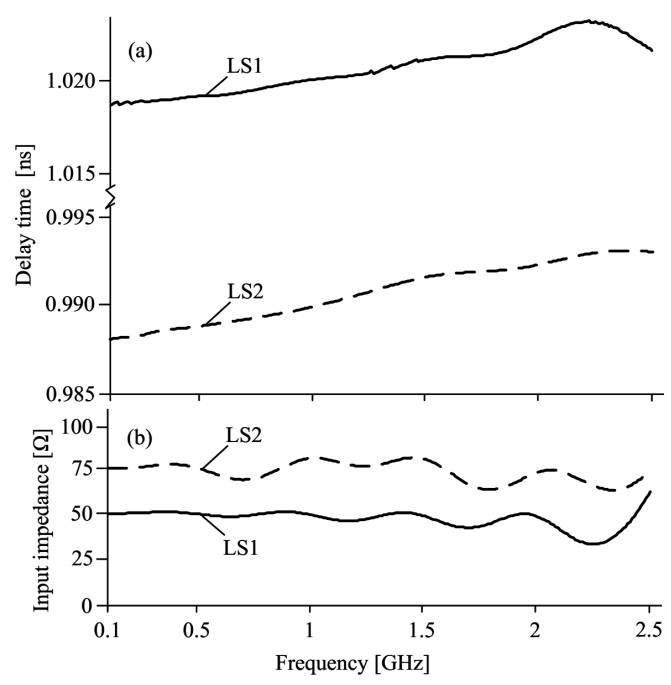

Fig. 5. Frequency response of phase delay time and input impedance of synthesized meander slow-wave systems with additional shields grounded at both edges LS1 and LS2.

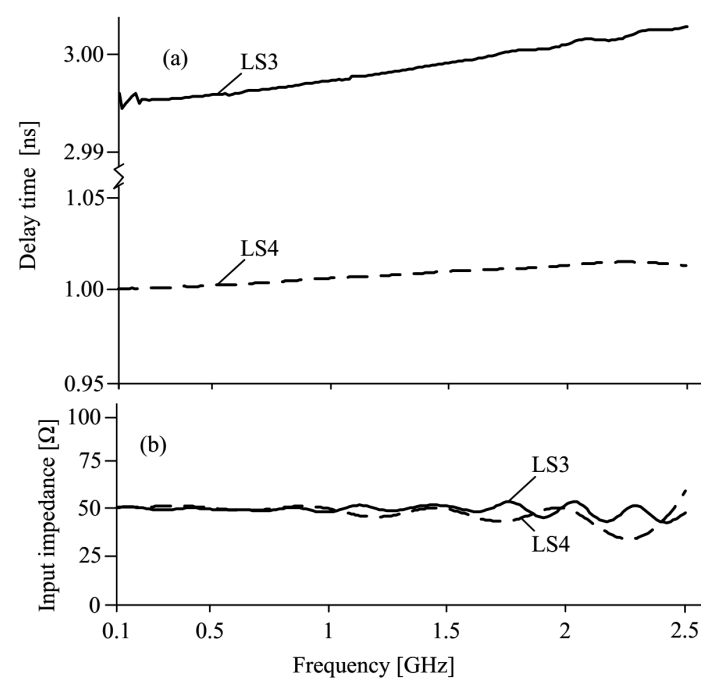

Fig. 6. As in Fig. 5, but for edges LS3 and LS4.

The meander slow-wave system with additional shields (LS3) has the lowest $\Delta F$ of $\mathrm{PB}$ because the constant delay time $t_{\mathrm{d}}$ is three times higher in (LS3) than in other meander slow-wave systems with additional shields. Also the dispersion in this type of meander slow-wave system with additional shields (LS3) is also higher than in other meander slow-wave systems with additional shields.

1 nanosecond delay time and $50 \Omega$ input impedance are obtained when the $\mathrm{RO} 3006^{\mathrm{TM}}$ material of dielectric substrate is replaced by FR4, the width $w_{1}$ and the length $2 A$ of meander conductor and the width $w_{2}$ of additional shield increase and the gap $s$ between adjacent strips of meander conductor decrease (LS1 and LS4). The width $\Delta F$ of $\mathrm{PB}$ decrease about $2 \%$ in this case. Frequency characteristics of the phase delay time and the input impedance of synthesized MSSs (LS1-LS4) are also presented in Fig. 5 and Fig. 6.

\section{Conclusions}

The algorithm of parametric synthesis of meander slow-wave systems with additional shields grounded at both ends has been concluded.

The appropriateness of parametric synthesis algorithm of MSSs with additional shields grounded at both ends was verified by performing synthesis of four different MSSs models.

Results show that the change of length of the meander conductor and the relative permittivity of substrate has the greatest impact on the width of the pass-band of the system. Width of the pass-band increases by about $1.36 \%$ as previously mentioned parameters values decreased 1\%. Changes of width of meander conductor, width of the gap between adjacent meander conductors and the thickness of the dielectric substrate has a significant impact on width of the pass-band. The width of the pass-band increases by $0.68 \%$ when the above-mentioned values of parameters decrease by $1 \%$. Changing the width of the additional screen does not affect the width of passband.

\section{References}

[1] W.S. Chang, C.Y. Chang, IEEE Trans. Microwave Theory Techn. 60, 11 (2012).

[2] M. Sumathy, L. Christie, S.K. Datta, L. Kumar, in: Proc. IEEE 13th Int. Vacuum Electronics Conf., IEEE, Monterey (CA) 2012.

[3] C.T. Chen, Appl. Mech. Mater. 241-244, 698 (2013).

[4] A. Kosi, M. Solar, J. Sci. Industr. Res. 69, 8 (2010).

[5] P. Vikram, H.V. Kumaraswamy, R.K. Manjunath, Int. J. Adv. Res. Comput. Commun. Eng. 4, 8 (2015).

[6] O. Diop, F. Ferrero, A. Diallo, G. Jacquemod, C. Laporte, H. Ezzeddine, C. Luxey, in: IEEE Int. Workshop on Antenna Technology, Ed. H. Zhang, IEEE, Tucson (AR) 2012.

[7] S. Lin, H. Cui, L. Wu, W. Wang, X. Sun, IET Microwaves Antennas Propagat. 6, 1 (2011).

[8] K.W. Hsu, W.H. Tu, Electron. Lett. 48, 15 (2012).

[9] J. Wu, J. Lou, M. Li, G. Yang, X. Yang, J. Adams, N.X. Sun, IEEE Trans. Microwave Theory Techn. 60, 6 (2012).

[10] S. Štaras, R. Martavičius, J. Skudutis, V. Urbanavičius, V. Daškevičius, Wide-Band Slow-Wave Systems: Simulation and Application, Taylor and Francis Group, New York 2012.

[11] E. Metlevskis, R. Martavičius, Electron. Electr. Eng. 20, 4 (2014).

[12] S. Zhang, Y.P. Zhang, Antennas Propagat. Mag. 53, 6 (2011).

[13] Y. Umayahara, Y. Ishikawa, Glass Ceramics Dielectric Material and Sintered Glass Ceramics, USA Patent, 6649550, US 09/988,023 (2003).

[14] E. Metlevskis, Ph.D. Thesis, Dept. Elect. Eng., Technika, Vilnius 2014. 